cause the urine to be secreted of a deeper colour than usual, and the solid matters consequently in considerable excess. In several diseases the same thing may happen. In pneumonia, several dyspeptic and febrile affections, when the urine becomes highly concentrated, it is of frequent occurrence, and depends upon a diminishing secretion of water only. In Typhus, Dr. Parkes found as much as 883 grains in twenty-four hours, and in a case of pyæmia, Vogel detected 1240 grains or nearly three ounces of urea within the same period. A case is recorded in the third volume of the Medico-Chirurgical Transactions, by Dr. Bostock, of a patient, a female, discharging five quarts of urine daily of a specific gravity of 1.034 , not saceharine, which on analysis was found to contain nine ounces and a half of solids, seren ounces and a half being urea, and two ounces salts.

This would be rather more than six times the average quantity for a healthy man. The patient recovered completely under the use of chalybeates.

The condition of the urine I have described, differs materially from all these cases: 1st, In the more or less persistent character of this secretion; 2nd, In the relative as well as absolute quantity of urea, as compared with the other ingredients, the quantity of water being normal, or only in slight excess. They correspond, therefore, with the first form alluded to by Prout, excess of urea without diuresis.

Sometimes the quantity of water, together with the urea and other products of the urine, are simultaneously increased. Excess of urea with diuresis: in such cases the quantity of urine discharged is described by Dr. Prout as being excessive. The quantity of renal uren in any given specimen may under such circumstances be relatively less than in health, but owing to the increase in the quantity of the urine, absolutely much above the natural standard. Prout considered these affections to be of rare occurrence, so much so indeed, that, where he had seen one case of this last form of the disease, he had seen twenty cases of diabetes. Parkes says, "I have never seen a disease of this kind; all the cases of excessive urea $I$ have seen have been either connected with pyrexia, or with some peculiarity of diet, excess of nitrogenous substances, or have been examples of diabetes insipidus, with excess of urea." Willis, who in his valuable work devotes several pages to its consideration, seems only to have noticed the second and more uncommon form of the disease. Most of the more recent cases I have read of, independent of the cutaneous affections with which both of my cases were associated, differ in some other respects.

The case mentioned by Dr. Sieveking in the Journac for June 1865, was 53 years of age, had jaundice, diarrhœa, and was otherwise extremely nervous and debilitated. Prout's cases all occurred in middle aged men, of thin, spare habits, and one likewise unusually nervous and depressed. Dr. Prout only met with one instance in the female. The cases recorded by Dr. Parkes and Roberts were also men of about 50. The daily flow of urine in Parkes's case amounted to ninety-six fluid ounces, in that of Dr. Roberts only thirty-four ounces, and the quantity of urea was never more than five hundred and fifty grains daily. Dr. Handfield Jones, in the October number of the Journal for 1861, under the title " Baruria," gives six additional cases, five males and one female. Three were under 25 years of age, the others between 45 and 50.

They were all persons evidently much out of health, and suffering from a variety of anomalons nerve symptoms; one was epileptic and died of dementia, the others yielded apparently to treatment.
The question of most importance to determine in connexion with the cases I have recorded, is the pathological relation of this condition of the urine to the cutaneous affection from which they both suffered; and, granting this supposed connexion, to trace the organ or function at fault in its production. The first case seemed to show there was a very obvious connexion between the two phenomena. By cutting off the supply of nitrogen from his food, in the instance of the young man first noticed, the density of the urine was immediately reduced, and the disease appeared to yield in a very striking way, but returned on his assuming his ordinary diet of meat once or twice daily, showing, apparently, as if a portion at least of the nitrogen taken as food is converted directly into urea in the blood, and eliminated by the kidney without becoming fixed in the general textures of the body. It would hardly be safe, however, to build up a theory of this disease from a single isolated case, as the main facts are unfortanately negatived in the second case. Similar restrictions, perseveringly carried out for many weeks, producing no such corresponding results.

This over-production, if I may use such an expression, of one of the most important and essential constituents of the urine, can have, I presume, but three sources. 1. It may originate from some defective or perverted action of the primary assimilating processes of digestion or chylification, whereby, as I have already stated, a portion of the alimentary materials are at once transformed into urea in the blood. 2. From that mysterious and more distant function of the animal economy by which the old and used up materials are unceasingly being carried away, and new ones deposited, and known to physiologists as the hystolitic process of disintegration, or metamorphosis of tissue; this is probably the main source of $c$ urea in a healthy individual. 3. From that less probable and scarcely recognised channel, a morbid or excited condition of the kidney itself, analogous, perhaps, to what some of the older writers regarded as a state of erythism of the organ, but which more advanced pathologists would, with perhaps greater propriety, call paresis of the renal plexus of nerves, with its at tendant results, dilatation of the bloodvessels and consequent increase of blood, and when there is more blood sent to an organ than usual, we may expect temporary increase of function. This is probably the condition of the kidney in cases of diabetes insipidus, and some nervous affections, in which such an enormous quantity of water is sometimes discharged, and which is often so effectually controlled by opium. In either case, we should probably have an abnormal quantity of urea very constantly circulating in the blood, spoiling, just as an excess of water is known sometimes to do, the red particles, and thus unfitting this vital stream for the healthy performance of nutrition, upon the due integrity of which the physiological well being of the individual must depend.

\section{ETHER-SPRAY IN THE REDUCTION OF HERNIA.}

Bу A. B. Steele, M.R.C.S.E., Liverpool.

THe account of Dr. Barclay's case, published on the 20 th ult, induces me to mention, that some months ago $I$ used the ether-spray with success in the reduction of a rather large inguinal hernia in a boy about 4 years old, who had worn a truss from early infancy. The hernia had remained down sufficiently long to cause vomiting and other early symptoms of stran. gulation.

Having persevered with the taxis in the ordinary 521 
way for some time, I applied the ether-spray, and ultimately succeeded in returning the bowel. I did not carry the frigorific effect so far as to produce complete congelation and blanching of the surface; but short of that condition, which might possibly have been injurious, the excessive coldness materially aided in the reduction of the hernia, and in all probability saved the patient from a capital operation.

While on the subject, I wish to communicate my experience with different kinds of ether. I have tried the ether sold by Mr. Robbins, that manufactured by Howard, and also methylated ether, and can discover no difference of effect in either of the three.

It is desirable that attention should be carefully directed to the relative efficiency of ether obtained from various sources; becuuse if, as I strongly suspect, it be found that the only essential matter is the rapidity of evaporation, as determined by specific gravity, the successful use of methylated ether, at a fourth of the price of other ethers, will be no small boon to those who desire to avail themselves of Dr. Richardson's valuable discovery in many cases where the expense of material falls upon the practitioner.

My first experiments with the ether-spray were uncertain, and often unsatisfactory ; and, on appealing to Dr. Richardson for an explanation, was told by him that it was all a question of ether, and that none except that sold by Robbins could be depended upon. Subsequent experience, however, has led me to believe that my previous want of success depended partly on some defect in the instrument, the wire in the nozzle having become encrusted with a deposit, and partly perhaps upon my own want of skill in manipulating. I find that I can now freeze the skin with ether prepared from methylated spirit quite as readily as with that sold by Mr. Robbins.

In the early days of chloroform, great stress was laid upon the importance of the purity of the chloroform; and some people believed that the right article could be obtained nowhere except from Duncan and Flockhart of Edinburgh.

I have used extensively, and for a long period, chloroform made with methylated spirit, which I find produce precisely the same results as any other; and I believe it is chemically and therapeutically identical with that prepared with alcohol, the only difference between the two being that one is more than double the price of the other.

\section{NOTES FROM PRACTICE.}

\section{By J. Birchenall, Esq., Macclesfield.}

IN forwarding the subjoined notes for insertion in the British Medical Journal, I would simply premise that the allusions therein contained are not designed to convey the slightest imputation on the professional competency of the gentlemen concerned.

As I have myself a reasonable share of the mental infirmities of our common humanity, I have learnt to be very chary in my reflections upon others; and I have lived long enough to know that, as the wisest of men are sometimes at fault, a point of practical importance may incidentally be overlooked even by those whom we are accustomed justly to regard as among the most cautious and discriminating of our medical brethren.

The cases occurred many years ago, and I write from memory, but can vouch for the accuracy of the reports.

CASE r. Rev. Mr. - , residing in a populous town in Yorkshire, was in the act of raising a teakettle full of boiling water from the fire, when it slipped from his hand, and fell upon his foot, which wasc. thereby severely bruised and scalded.

When the injury to the skin was repaired, he found that he could not walk without considerablepain; but, as the medical gentleman in attendancec (who was a respectable practitioner) regarded the음 case as a purely hyperæsthetic condition, it was $\frac{\bar{\sigma}}{\bar{\sigma}}$ treated accordingly.

After the lapse of some weeks, as there was no으 improvement, Mr. - , at the instance of some of his friends, visited a hydropathic establishment, but, returning unrelieved, he subsequently took the ad-o vice, at different intervals, of two or three eminent sur- $\overrightarrow{-}$ geons in succession, who thought that the inconveni- $\vec{\omega}$ ence complained of was owing to chronic inflamma- o tion of the tarsal articulating surfaces, or was the $\frac{0}{3}$ result of an arthritic diathesis. Several months had. intervened during the treatment suggested without? any relief, when Mr. - being on a visit to some of his friends in this town, who were my patients, I waso requested to look at the case. I found the innercr cuneiform bone, which was the seat of pain, enlarged; pressure on the part giving to the patient a sharpo pricking sensation; and, by grasping the scaphoid bone with one hand, and by forcible pressure with $\overrightarrow{0}$ the other, attempting a rotatory movement of the metatarsal bone of the great toe upon its tarsal arti-o culation, I detected a distinct crepitus in the enlarged cuneiform bone.

After submitting to the patient and his friends, that six or eight weeks of perfect rest would be re-은 quired for the successful treatment of the case, $\vec{c}$ arrangements were made for this purpose. The fogto was closely enveloped in a starched bandage, over. which a roller was applied, and the limb maintained in a state of perfect repose. At the end of six weet; as the tenderness was gone, an elastic sock was sutbstituted for the previous appliances, and gradual $\vec{e} \cdot \overline{\bar{x}}$ ercise allowed.

Mr. - returned home in two or three weeks more, 응 nothing further being required but the use, for a time, of a boot of easier adaptation than ordinary, $\overrightarrow{\vec{A}}$ and he has since prosecuted his duties with unremit- 3 ting activity, without the slightest inconvenience.

CASE II. Rev. Mr. B., aged 63, of robust frame, of nervous sanguine temperament, fair and florid complexion, and of perfectly regular and active habits, came to reside in this town about fifteen years ago. His health, previously uniformly good, had been failing for some time. He complained of lassitude, $\bar{\sigma}$ weariness on slight exertion, with loss of appetite, 3 . and occasional nausea. The head was free from pain, the intellect clear; there was no vertigo, or any disturbance in the organs of special sense; no cough or dyspncea; the action of the heart was 음 normal; the belly soft, no tenderness on pressure throughout its whole extent; no glandular or other $\mathbb{N}$ enlargement; the bowels a little torpid; the tongue $D$ pretty clean and moist; but the sleep was disturbed; the spirits, ordinarily buoyant. began to droop; and there was a brooding presentiment that " this sick- N ness would be unto death."

In the absence of all indications of positive disease, $\omega$ I was induced to regard the case as one of purely climacteric decay, and had recourse mainly to hygi-co enic means for its relief.

There was one inconvenience of which my patient $\stackrel{\$}{\mathscr{S}}$ early complained; namely, a difficulty in emptying the bladder. The urine was clear, and free from de- 0 posits, flowed in a full stream, after an effort of a few $\bar{O}$ seconds, and was discharged in moderate quantity; $\mathbb{\mathbb { D }}$ but there was always a lingering consciousness that $\frac{?}{9}$ the relief was incomplete. I expressed a suspicion $\triangle$ that there might be some enlargement of the pro- $\bar{\sigma}$ 